2017-10-01

\title{
The Evaluation of the Effect of Strain Limits on the Physical Properties of Magnetorheological Elastomers Subjected to Uniaxial and Biaxial Cyclic Testing.
}

\author{
Dave Gorman \\ Technological University Dublin, david.gorman@tudublin.ie \\ Niall Murphy \\ Technological University Dublin, niall.murphy@tudublin.ie \\ Ray Ekins \\ Technological University Dublin, ray.ekins@tudublin.ie
}

See next page for additional authors

Follow this and additional works at: https://arrow.tudublin.ie/engschmecart

Part of the Physics Commons

\section{Recommended Citation}

Gorman D., Murphy N.,Ekins R., et al. (2017). The evaluation of the effect of strain limits on the physical properties of Magnetorheological Elastomers subjected to uniaxial and biaxial cyclic testing. International Journal of Fatigue 103: 1-4. https://doi.org/10.1016/j.ijfatigue.2017.05.011

This Article is brought to you for free and open access by the School of Mechanical and Design Engineering at ARROW@TU Dublin. It has been accepted for inclusion in Articles by an authorized administrator of ARROW@TU Dublin. For more information, please contact arrow.admin@tudublin.ie, aisling.coyne@tudublin.ie, gerard.connolly@tudublin.ie.

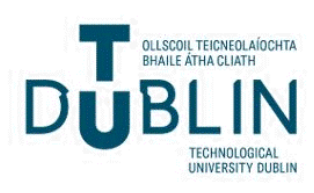




\section{Authors}

Dave Gorman, Niall Murphy, Ray Ekins, and Stephen Jerrams 
Title: The evaluation of the effect of strain limits on the physical properties of Magnetorheological Elastomers subjected to uniaxial and biaxial cyclic testing.

\title{
Authors and Affiliations
}

Dave Gorman, Niall Murphy, Ray Ekins, and Stephen Jerrams

Dublin Institute of Technology Dublin 1

Corresponding Author Dave Gorman david.gorman@dit.ie

\begin{abstract}
Magnetorheological Elastomers (MREs) are "smart" materials whose physical properties are altered by the application of magnetic fields. In a previous study by the authors [1], variations in the physical properties of MREs have been evaluated when subjected to a range of magnetic field strengths for both uniaxial and biaxial cyclic tests. By applying the same magnetic field to similar samples, this paper investigates the effect of both the upper strain limit and the strain amplitude on the properties of MREs subjected to cyclic fatigue testing. As the magnetorheological (MR) effect is due to the dipole-dipole interactions of the magnetic particles in an MRE [2], it is expected that the larger the upper strain limit, the lower the overall MR effect will be. This is investigated by varying the upper strain limit between tests while keeping the magnetic field applied during testing at selected cycles constant between tests. To investigate if the MR effect is only dependent on the upper strain limit and the magnitude of the applied magnetic field during cyclic testing, the tests are repeated with the same upper strain limits and applied fields but with reduced strain amplitude.
\end{abstract}

\section{Keywords}

Magnetorheological Elastomers; Magnetic fields; Uniaxial tension; Biaxial bubble inflation; Natural Rubber; Fatigue

\section{Introduction}

Magnetorheological Elastomers (MREs) are classified as smart materials that undergo changes in their physical properties which are observed as an increase in modulus when a magnetic field is applied to an MRE [3]. The increase in the modulus is caused by the ferromagnetic particles which are added to the elastomer during the curing process, tending to align with the applied magnetic field. The alignment occurs due to the applied field causing dipole-dipole interactions between the particles which move to screen each other from the field and adopt a minimum energy configuration [2].

MREs can be described as either isotropic or anisotropic depending on whether a magnetic field was applied during the curing process. Isotropic MREs have an almost homogeneous distribution of magnetic particles and are cured without the presence of a magnetic field, whereas anisotropic MREs contain aligned particle chains formed by the alignment of the magnetic particles with an external magnetic field applied during the curing process [4]. While anisotropic MREs provide greater MR properties when the magnetic field is applied parallel to the particle chains [4], the presence of the magnetic field during the curing process introduces an extra variable into the experimental results obtained from cyclic testing. For this reason isotropic MRE samples were used in the tests to ensure that any measured changes in their properties can be fully attributed to changes in test conditions and not 
changes in the sample particle distribution due to variations in the magnetic field applied during the curing process[1].

While the influence of the applied magnetic field on the MR effect of MREs has been presented in many reports [2-11] there is a lack of comparable data for similar MRE samples tested under different strain conditions using the same applied magnetic fields. In particular, experimental results do not exist for MREs cycled at high strain for both uniaxial and biaxial conditions $[1,11]$

The focus of this research is to investigate the influence of strain limits on the MR effect for MREs under cyclic uniaxial and biaxial loading. This is achieved by testing MREs under both uniaxial and biaxial loading conditions with similar strain limits and with the same applied magnetic field. The tests were repeated with the same maximum strain but lower strain amplitudes. As the effective strain in biaxial tension for a specific strain limit is much higher than in the uniaxial case, a direct comparison of the properties (modulus) is impossible but trends can be compared[1].

As the MR effect is due to the to the interaction of magnetic particles [2] and this interaction is inversely proportional to the square of the distance between the particles [12], it would be expected that the upper or maximum strain rather than the strain amplitude would predominantly govern the overall MR effect.

\section{Apparatus and Materials}

\subsection{Magnetorheological Elastomers}

The MRE samples used in all tests reported in this paper are carbon black filled natural rubber based MREs from the same sample batch used to investigate the effect of magnetic flux density on the MR effect in an earlier study [1]. A number of previous studies by other researchers [7-9], focused on NR based MREs. NR was chosen due to its superior physical (modulus) and a fatigue property, which is important for isolating the MR effect as with softer rubbers, such as silicone or urethane, the change from cycle to cycle may be attributable to fatigue effects in the matrix material.

The test method is described in the previous study by the authors [1] with the same sample dimension for both the uniaxial and biaxial tests being used.

\subsection{The magnetic field}

Flux density values generated by the electromagnetic arrays are given for the centre point of the array without the presence of a magnetic sample. For the uniaxial tests this value has a maximum deviation of $15 \%$ of the stated value at a displacement of $20 \mathrm{~mm}$ from the centre point. For the biaxial bubble inflation tests, the variation in the flux density over the area in which the vision system records the stress strain data is $5 \%$ of the stated flux density value. The deviations reported are inevitably a characteristic of magnetic fields in air due to the $1 / \mathrm{r}^{2}$ relationship [13]. All magnetic fields applied in this study to both the uniaxial and biaxial tests were generated by the same electromagnetic array used and outlined in a previous study by the authors[1].

\section{Testing methods}

3.1 Uniaxial tensile fatigue tests 
Uniaxial tensile fatigue tests were performed on the isotropic NR MREs with the strain and magnetic flux density applied in the same directions as the previous study by the same authors which investigated the MR response of the material samples of $70 \mathrm{~mm} \times 20 \mathrm{~mm} \times 1 \mathrm{~mm}$ under different applied magnetic flux densities[1].

As with the previous investigation into the effect of the applied magnetic flux density on the overall MR effect [1], the magnetic fields where cycled between the off and on positions every 50 cycles starting with no flux applied for the first 50 cycles. However, the flux densities applied in all uniaxial fatigue tests in this paper were constant between tests with the applied strain conditions being varied for each test.

\subsection{Equi-biaxial bubble inflation tests.}

The bubble inflation tests were carried out on the DYNAMET equibiaxial bubble inflation test machine developed in the Dublin Institute of Technology by Murphy et al $[14,15]$ and further developed by Johnson et al [16, 17]

As with the uniaxial tests, the magnetic field was cycled between its on and off positions every 20 cycles at the same cycle numbers as the previous investigation (off for cycles 70-89, on for cycles 90-109) [1]. The flux density was held constant for all bubble inflation tests in this paper however, the strain conditions where varied between the different bubble inflation tests.

The focus of this study is to determine the effect of altering strain limits on the MR effect.

\section{Results}

\subsection{Uniaxial tensile test results}

All tests on MREs reported in this study were for cyclic loading between fixed strain limits $(\Delta \mathrm{L})$. The MR effect was calculated as the increase in the average modulus for a block of 50 cycles using the final 200 cycles of a uniaxial test. The later cycles of the test where chosen for analysis of the MR effect as the early cycles show diminishing maximum stress values due to the Mullins effect [18]. It was observed that the material properties had stabilized by the latter stages of the tests and therefore any change in properties of the material was due to the applied magnetic flux density. The error bars represent the standard error (mathematical error on the mean due to sample size). The stepped increase in modulus, visible at the 360th cycle on the $\mathrm{x}$-axis in figure 1 , is from $1.325 \mathrm{MPa}$ to $1.413 \mathrm{MPa}$. This is an increase of approximately $6.5 \%$ in the average modulus of the 50 cycle block. This corresponds with the field being switched on at cycle 360 . 


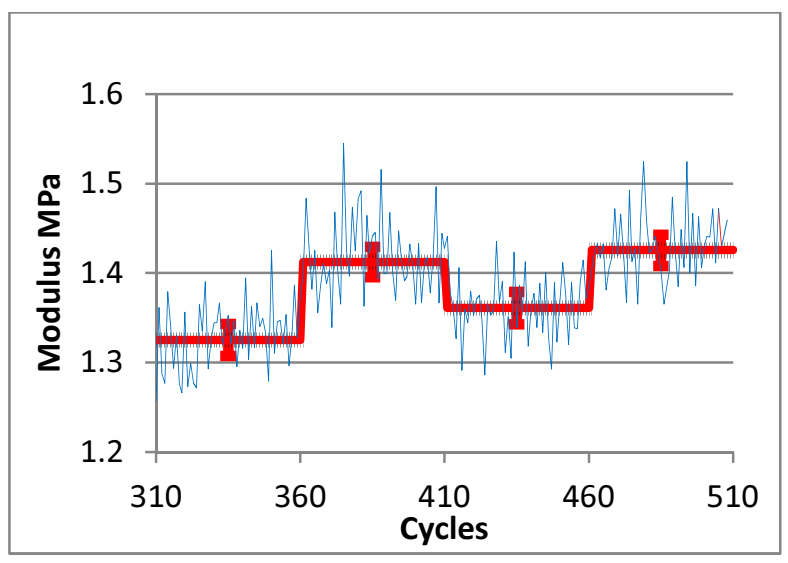

Fig 1 Average Modulus v Cycles in uniaxial testing at a flux density of $206 \mathrm{mT}$ for a

strain amplitude of $0.04-0.08$

The results presented in figure 2 are for a similar test to those shown in figure 1 but with higher strain amplitude applied (0.04-0.57). From the data presented in figure 2, the increase in the block average modulus at cycle 350 was from $3.562 \mathrm{MPa}$ to $3.591 \mathrm{MPa}$. This is an increase of approximately $0.8 \%$ in the average modulus of the 50 cycle block. This corresponds with the field being switched on at cycle 350 . On comparing the data in figures 1 and 2 it is clear that as the strain increases, the MR effect decreases. This was to be expected as the particles were further apart from each other at higher strain, reducing screening effects when compared with lower strain conditions.

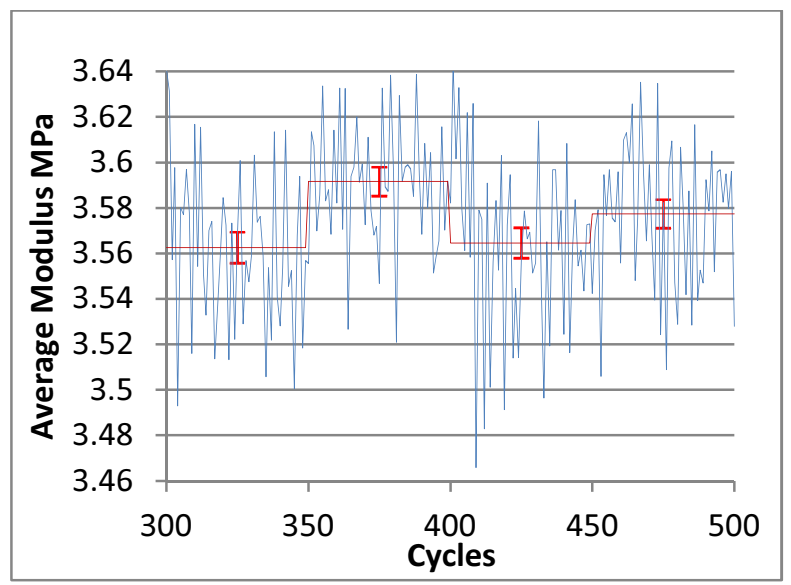

Fig 2 Average Modulus v Cycles for uniaxial data at a flux density 206mT for a

strain amplitude of $0.04-0.57$

A further test was carried out with strain limits from 0.47-0.57 hence having the same upper strain limit as that shown in figure 2 but with a lower strain amplitude. The increase in the block average modulus observed in the test presented in figure 3 at cycle 350 is from $3.699 \mathrm{MPa}$ to $3.711 \mathrm{MPa}$, an approximate increase of $0.32 \%$. The actual modulus is higher than that observed in figure 2 as the modulus is a function of strain and without the inclusion of the lower strain values in figure 2 , the average will increase. The increase in modulus in figure 3 is similar to that shown in figure 2 and lower than the increase observed in figure 1 which suggests that it is the maximum strain limit which determines the overall MR effect and not the strain amplitude. 


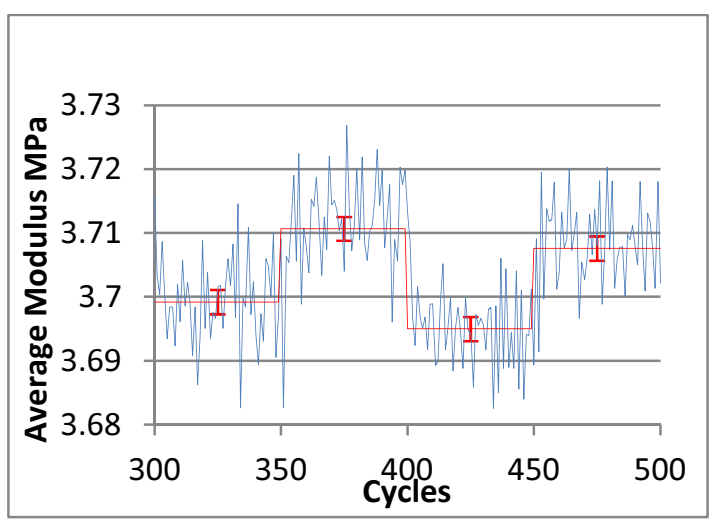

Fig 3 Average Modulus v Cycles for uniaxial data at a flux density 206mT

for a strain amplitude of $0.47-0.57$

\subsection{Biaxial bubble inflation test results}

Samples were also tested under cyclic biaxial loading by bubble inflation of $1 \mathrm{~mm}$ thick discs between different strain limits with the same magnetic flux densities applied throughout the equi-biaxial tests. These tests were carried out using the same number of cycles, and with the same applied field conditions as the previous investigation into the dependency of the MR effect on the applied magnetic flux density[1]. No results were processed during the first 90 cycles to minimise the levels of Mullins effect on the samples as was the case for the first 300 cycles in the uniaxial tests.

Figure 4 shows the final 80 cycles for a low strain (0.0-0.1) biaxial bubble inflation test at a flux density of $198 \mathrm{mT}$. The increase in the modulus recorded at cycle 90 was from $3.066 \mathrm{MPa}$ to $3.132 \mathrm{MPa}$ corresponding to an increase of $2.2 \%$ in the block average modulus when the flux density is applied to the sample.

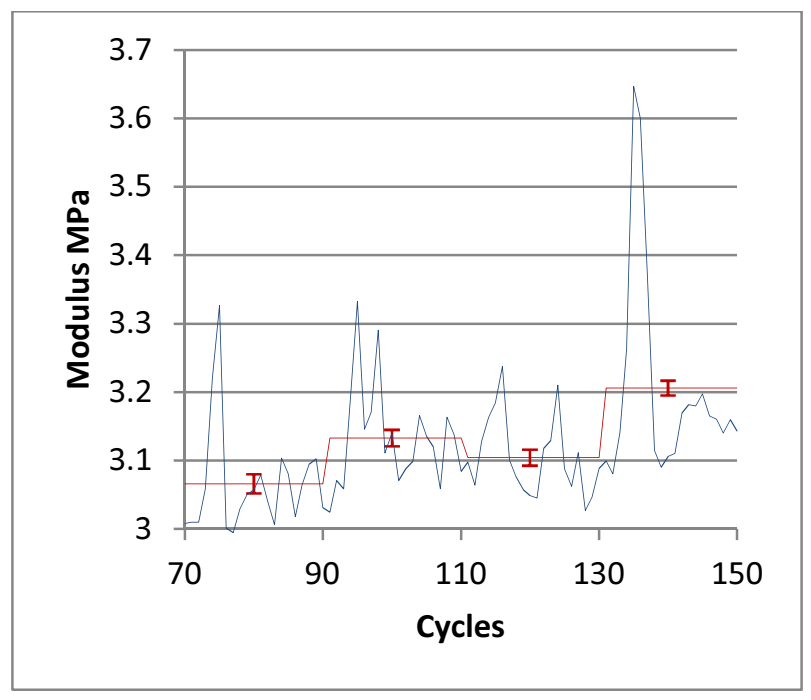

Fig 4 Average Modulus v Cycles for biaxial data at a flux density of $198 \mathrm{mT}$ and a

strain amplitude of $0-.1$

The data shown in figure 5 is for a similar bubble inflation test with the same magnetic flux density conditions as those presented in figure 4 but with a higher strain applied (0-0.5). 


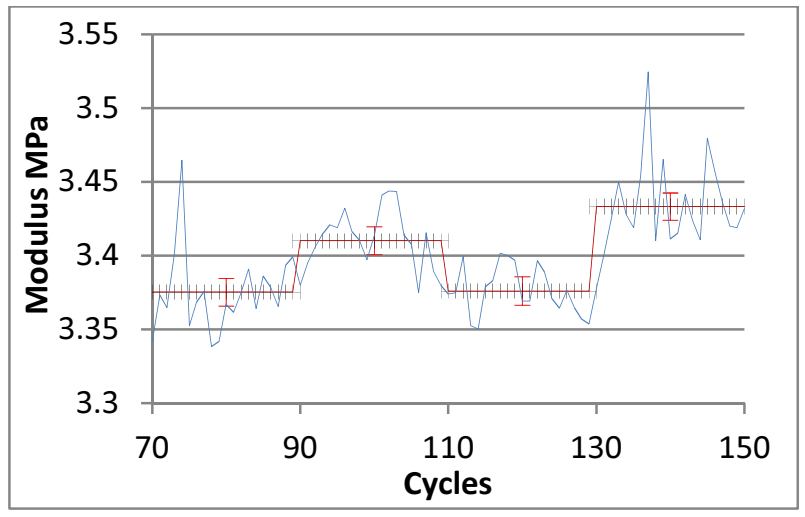

Fig 5 Average Modulus v Cycles for biaxial data at a flux density 198mT

and a strain amplitude of 0-0.5

The increase in the block average modulus due to the application of the magnetic flux density at cycle 90 is from $3.375 \mathrm{MPa}$ to $3.410 \mathrm{MPa}$ which is an increase of approximately $1 \%$ which is a just under half of that for the low strain test shown in figure 4.

The data in figure 6 is for a biaxial test with identical magnetic flux density conditions to those presented in figures 4 and 5 but in this case at the higher strain and a lower strain amplitude applied (0.4-0.5) similar to the uniaxial test shown in figure 3.

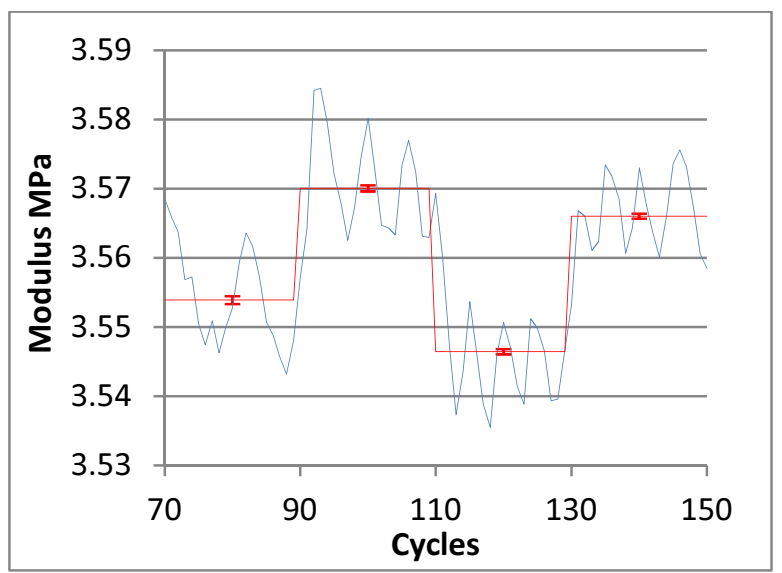

Fig 6 Average Modulus v Cycles for biaxial data at a flux density 198mT and a strain amplitude of 0.4-0.5

It can be observed from figure 6 the MR effect detected at cycle 90 is from $3.553 \mathrm{MPa}$ to $3.570 \mathrm{MPa}$, an increase of approximately $0.4 \%$. This is a similar result to that obtained for the uniaxial data, this is of a similar magnitude to the high strain, high strain amplitude tests but lower that the MR effect observed in the low strain amplitude low strain test cases.

\section{Conclusions}

From the results shown in figures 1-3 for uniaxial test conditions and those in figures 4-6 for equi-biaxial bubble inflation tests, it is demonstrated that overall strain and not strain amplitude has the greatest influence on the magnetic response of an MRE sample subjected to a magnetic field and cyclic loading. 
It is not possible to give a direct comparison between uniaxial and biaxial data even with samples from the same batch and with the same electromagnetic array used to provide the magnetic field, as the stress-strain relationship for uniaxial and biaxial strain are different. However, trends in the relationship between MR effects and the applied strain conditions can be compared.

In both series of tests, the largest MR effect was observed for low strain tests. This is due to the physical phenomenon that is responsible for any MR effect; the movement of magnetic particles to align with the applied magnetic field and adopt a minimum energy configuration [12]. As magnetic interactions are proportional to the square of the distance between the particles [13], this result is as expected.

For the high strain conditions using both test methods, a similar lower MR effect was observed when both high and low strain amplitudes were applied with the high strain amplitude cases showing a slightly higher MR response. This can be attributed to the fact that in the high strain amplitude tests, the samples spend a portion of the cycle at a lower strain value which reduces the distance between the particles and as the MR effect is calculated using an average modulus, these data points are included in the calculation of that MR effect. Therefore, for any MRE based product used over a large strain range, the MR effect will be dependent on the strain and a greater change in modulus will be achieved for the same applied flux density for the portion of the time it is operating under low strain conditions by comparison with high strain conditions.

For any high strain amplitude applications, the MR effect will not be constant over the entire strain range. This fact highlights the importance of stating the strain limits for any reported MRE test results.

While the high strain, low strain amplitude tests, show the lowest MR effect, they can provide useful data on the high strain performance of MREs and provide the only way of accurately determining the strain value at which the MR effect is no longer present. With high strain, high strain amplitude tests, the low strain regions of the cycles during testing will cause an MR effect to be detected. As the MR effect is indicated by an increase in the average modulus, an increase in modulus will be present even if the MR effect is zero for the high strain portion of the cycle.

\section{Further Work}

It is proposed to carry out further tests under both uniaxial and biaxial conditions to graphically represent a mathematical relationship between MR effects and strain limits.

\section{Acknowledgements}

This work was carried out in the School of Mechanical \& Design Engineering, Dublin Institute of Technology. 
1. Gorman, D., et al., The evaluation and implementation of magnetic fields for large strain uniaxial and biaxial cyclic testing of Magnetorheological Elastomers. Polymer Testing, 2016. 51: p. 74-81.

2. Stepanov, G.V., et al., Effect of a homogeneous magnetic field on the viscoelastic behavior of magnetic elastomers. Polymer, 2007. 48(2): p. 488-495.

3. Boczkowska, A. and S. Awietjan, Smart composites of urethane elastomers with carbonyl iron. Journal of Materials Science, 2009. 44(15): p. 4104-4111.

4. Varga, Z., G. Filipcsei, and M. Zrínyi, Magnetic field sensitive functional elastomers with tuneable elastic modulus. Polymer, 2006. 47(1): p. 227-233.

5. Boczkowska, A., et al. Effect of the processing conditions on the microstructure of urethane magnetorheological elastomers. 2006. San Diego, CA, USA: SPIE.

6. Boczkowska, A., et al., Image analysis of the microstructure of magnetorheological elastomers. Journal of Materials Science, 2009. 44(12): p. 3135-3140.

7. Chen, L., et al., Investigation on magnetorheological elastomers based on natural rubber. Journal of Materials Science, 2007. 42(14): p. 5483-5489.

8. Deng, H.X. and X.L. Gong, Adaptive Tuned Vibration Absorber based on Magnetorheological Elastomer. Journal of Intelligent Material Systems and Structures, 2007. 18(12): p. 12051210.

9. GONG, X.L., L. CHEN, and J.F. LI, STUDY OF UTILIZABLE MAGNETORHEOLOGICAL ELASTOMERS. International Journal of Modern Physics B, 2007. 21(28n29): p. 4875-4882.

10. McIntyre J, Jerrmans S, and Alshuth T, Magnetorheological Elastomers Developemt of MRES for automotive applications. Tire Technology International, 2013: p. 42-45.

11. Schubert, G. and P. Harrison, Large-strain behaviour of Magneto-Rheological Elastomers tested under uniaxial compression and tension, and pure shear deformations. Polymer Testing, 2015. 42: p. 122-134.

12. Grant, I.S. and W.R. Phillips, Electromagnetism1975: Wiley.

13. Maxwell, J.C., On Physical Lines of Force1862.

14. Murphy, N., et al. Determining multiaxial fatigue in elastomers using bubble inflation. in CONSTITUTIVE MODELS FOR RUBBER-PROCEEDINGS-. 2005. Balkema.

15. Murphy, N., J. Hanley, and S. Jerrams, A method of real-time bi-axial strain control in fatigue testing of elastomers. Constitutive Models for Rubber VII, 2011: p. 409.

16. Johnson, M., et al., The equi-biaxial fatigue characteristics of EPDM under true (Cauchy) stress control conditions. Constitutive Models for Rubber VIII, 2013: p. 383.

17. Johnson, M., et al., Equi-biaxial fatigue testing of EPM utilising bubble inflation. Polymer Testing, 2016. 53: p. 122-131.

18. Mullins, L. and N. Tobin, Stress softening in rubber vulcanizates. Part I. Use of a strain amplification factor to describe the elastic behavior of filler-reinforced vulcanized rubber. Journal of Applied Polymer Science, 1965. 9(9): p. 2993-3009. 